\title{
Rheumatology Around The World ... What is New?
}

\author{
EULAR Recommendations for the Management of Rheumatoid Arthritis with Synthetic \\ and Biological Disease-Modifying Antirheumatic Drugs: 2013 Update
}

\author{
Josef S. Smolen et al.
}

Ann Rheum Dis doi:10.1136/annrheumdis-2013-204573

In this article, the 2010 European League against Rheumatism (EULAR) recommendations for the management of rheumatoid arthritis (RA) with synthetic and biological disease-modifying antirheumatic drugs (sDMARDs and bDMARDs, respectively) have been updated. The 2013 update has been developed by an international task force, which based its decisions mostly on evidence from three systematic literature reviews (one each on sDMARDs, including glucocorticoids, bDMARDs and safety aspects of DMARD therapy); treatment strategies were also covered by the searches. The evidence presented was discussed and summarised by the experts in the course of a consensus finding and voting process. Levels of evidence and grades of recommendations were derived and levels of agreement (strengths of recommendations) were determined. Fourteen recommendations were developed (instead of 15 in 2010). Some of the 2010 recommendations were deleted, and others were amended or split. The recommendations cover general aspects, such as attainment of remission or low disease activity using a treat-to-target approach, and the need for shared decisionmaking between rheumatologists and patients. The more specific items relate to starting DMARD therapy using a conventional sDMARD (csDMARD) strategy in combination with glucocorticoids, followed by the addition of a bDMARD or another csDMARD strategy (after stratification by presence or absence of adverse risk factors) if the treatment target is not reached within 6 months (or improvement not seen at 3 months). Tumour necrosis factor inhibitors (adalimumab, certolizumab pegol, etanercept, golimumab, infliximab, biosimilars), abatacept, tocilizumab and, under certain circumstances, rituximab are essentially considered to have similar efficacy and safety. If the first bDMARD strategy fails, any other bDMARD may be used. The recommendations also address tofacitinib as a targeted sDMARD (tsDMARD), which is recommended, where licensed, after use of at least one bDMARD. Biosimilars are also addressed. These recommendations are intended to inform rheumatologists, patients, national rheumatology societies and other stakeholders about EULAR's most recent consensus on the management of RA with sDMARDs, glucocorticoids and bDMARDs. They are based on evidence and expert opinion and intended to improve outcome in patients with RA.

\section{Classification Criteria for Systemic Sclerosis}

\author{
van den Hoogen et al. \\ Arthritis \& Rheumatism, Vol. 65, No. 11, November 2013, pp. 2737-2747
}

Objective: The 1980 American College of Rheumatology (ACR) classification criteria for systemic sclerosis (SSc) lack sensitivity for early SSc and limited cutaneous SSc. The present work, by a joint committee of the ACR and the European League Against Rheumatism (EULAR), was undertaken for the purpose of developing new classification criteria for SSc. Methods: Using consensus methods, 23 candidate items were arranged in a multicriteria additive point system with a threshold to classify cases as SSc. The classification system was reduced by clustering items and simplifying weights. The system was tested by 1) determining specificity and sensitivity in SSc cases and controls with scleroderma-like disorders, and 2) validating against the combined view of a group of experts on a set of cases with or without SSc. Results: It was determined that skin thickening of the fingers extending proximal to the metacarpophalangeal joints is sufficient for the patient to be classified as having SSc; if that is not present, 7 additive items apply, with varying weights for each: skin thickening of the fingers, fingertip lesions, telangiectasia, abnormal nailfold capillaries, interstitial lung disease or pulmonary arterial hypertension, Raynaud's phenomenon, and SScrelated autoantibodies. Sensitivity and specificity in the validation sample were, respectively, 0.91 and 0.92 for the new classification criteria and 0.75 and 0.72 for the 1980 ACR classification criteria. All selected cases were classified in accordance with consensusbased expert opinion. All cases classified as SSc accord ing to the 1980 ACR criteria were classified as SSc with the new criteria, and several additional cases were now considered to be SSc. Conclusion: The ACR/EULAR classification criteria for SSc performed better than the 1980 ACR criteria for SSc and should allow for more patients to be classified correctly as having the disease. 


\title{
Treating Spondyloarthritis, Including Ankylosing Spondylitis and Psoriatic Arthritis, to Target: Recommendations of an International Task Force
}

\author{
Josef S Smolen et al. \\ Ann Rheum Dis. 2014; 73: 6-16.
}

Background: Therapeutic targets have been defined for diseases like diabetes, hypertension or rheumatoid arthritis and adhering to them has improved outcomes.Such targets are just emerging for spondyloarthritis (SpA). Objective: To define the treatment target for SpA including ankylosing spondylitis and psoriatic arthritis (PsA) and develop recommendations for achieving the target, including a treat-to-target management strategy. Methods: Based on results of asystematic literature review and expert opinion, a task force of expert physicians and patients developed recommendationswhich were broadly discussed and voted upon in a Delphi-like process. Level of evidence, grade and strength of the recommendations were derived by respective means. The commonalities between axial SpA, peripheral SpA and PsA were discussed in detail. Results: Although the literature review did not reveal trials comparing a treatto-target approach with another or no strategy, it provided indirect evidence regarding an optimised approach to therapy that facilitated the development of recommendations. The group agreed on 5 overarching principles and 11 recommendations; 9 of these recommendations related commonly to the whole spectrum of SpA and PsA, and only 2 were designed separately for axial SpA, peripheral SpA and PsA. The main treatment target, which should be based on a shared decision with the patient, was defined as remission, with the alternative target of low disease activity. Followup examinations at regular intervals that depend on the patient's status should safeguard the evolution of disease activity towards the targeted goal. Additional recommendations relate to extra-articular and extramusculoskeletal aspects and other important factors, such as comorbidity. While the level of evidence was generally quite low, the mean strength of recommendation was 9-10 (10: maximum agreement) for all recommendations. A research agenda was formulated. Conclusions: The task force defined the treatment target as remission or, alternatively, low disease activity, being aware that the evidence base is not strong and needs to be expanded by future research. These recommendations can inform the various stakeholders about expert opinion that aims for reaching optimal outcomes of SpA.

\section{Biomarkers in Vasculitis}

\author{
Monach, Paul A. \\ Current Opinion in Rheumatology: January 2014, Volume 26, Issue 1, pp. 24-30
}

Purpose of review: Better biomarkers are needed for guiding management of patients with vasculitis. Large cohorts and technological advances had led to an increase in preclinical studies of potential biomarkers. Recent findings: The most interesting markers described recently include a gene expression signature in CD8+ T cells that predicts tendency to relapse or remain relapse-free in antineutrophil cytoplasmic antibody (ANCA)-associated vasculitis, and a pair of urinary proteins that are elevated in Kawasaki disease but not other febrile illnesses. Both of these studies used 'omics' technologies to generate and then test hypotheses. More conventional hypothesis-based studies have indicated that the following circulating proteins have potential to improve upon clinically available tests: pentraxin-3 in giant cell arteritis and Takayasu's arteritis; von Willebrand factor antigen in childhood central nervous system vasculitis; eotaxin-3 and other markers related to eosinophils or Th2 immune responses in eosinophilic granulomatosis with polyangiitis (Churg-Strauss syndrome); and matrix metalloproteinase-3, tissue inhibitor of metalloproteinase-1, and CXCL13 in ANCA-associated vasculitis. Summary: New markers testable in blood and urine have the potential to assist with diagnosis, staging, assessment of current disease activity, and prognosis. However, the standards for clinical usefulness, in particular, the demonstration of either very high sensitivity or very high specificity have yet to be met for clinically relevant outcomes.

Egypt J Rheumatology and Clinical Immunology | Jan 2014 | Vol. 2 | Issue 1 


\title{
Efficacy of Aspirin for the Primary Prevention of Thrombosis in Patients with Antiphospholipid Antibodies: An International and Collaborative Meta-Analysis
}

\author{
Laurent Arnaud et al. \\ Autoimmunity Reviews, Volume 13, Issue 3, March 2014, pp. 281-291
}

\begin{abstract}
We performed a meta-analysis to determine whether aspirin has a significant protective effect on risk of first thrombosis among patients with antiphospholipid antibodies $(\mathrm{aPL}+)$. Observational and interventional studies identified from the Medline, Embase and Cochrane databases were selected if they assessed the incidence of first thrombosis in $\mathrm{aPL}+$ patients treated with aspirin versus those without. Pooled effect estimates were obtained using a random-effects model. Of 1211 citation retrieved, 11 primary studies (10 observational and 1 interventional) met inclusion criteria, including a total of 1208 patients and 139 thrombotic events. The pooled odds ratio (OR) for the risk of first thrombosis in patients treated with aspirin $(n=601)$ was 0.50 (95\% CI: 0.27 to 0.93$)$ compared to those without aspirin $(n=607)$, with significant heterogeneity across studies $\left(I^{2}=46 \%, p=0.05\right)$. Subgroup analysis showed a protective effect of aspirin against arterial (OR: 0.48 [95\%CI: $0.28-0.82]$ ) but not venous (OR: 0.58 [95\% CI: $0.32-$ 1.06]) thrombosis, as well as in retrospective (OR: 0.23 [0.13-0.42]) but not prospective studies (OR: 0.91 [0.521.59]). Subgroup analysis according to underlying disease revealed a significant protective effect of aspirin for asymptomatic aPL + individuals (OR: 0.50 [0.25-0.99]), for systemic lupus erythematosus (SLE) (OR: 0.55 [0.310.98]) and obstetrical antiphospholipid syndrome (APS) (OR: 0.25 [0.10-0.62]). This meta-analysis shows that the risk of first thrombotic event is significantly decreased by low dose aspirin among asymptomatic aPL individuals, patients with SLE or obstetrical APS. Importantly, no significant risk reduction was observed when considering only prospective studies or those with the best methodological quality.
\end{abstract}

Key Words: Aspirin; Thrombosis; Meta-analysis; Antiphospholipid antibodies

\section{Prednisone in Lupus Nephritis: How Much is Enough?}

\author{
Guillermo Ruiz-Irastorza et al. \\ Autoimmunity Reviews Volume 13, Issue 2, February 2014, pp. 206-214
}

\begin{abstract}
Objective: To assess the effectiveness and safety of a protocol using medium doses of prednisone to treat lupus nephritis. Methods: Patients receiving the 'Cruces-protocol cohort' (CPC) were paired 1:2 with patients from the 'historic cohort' (HC). The CPC received medium doses of prednisone combined with methyl-prednisolone pulses, hydroxychloroquine and immunosuppressive drugs, usually cyclophosphamide. The HC received cyclophosphamide and high-dose prednisone. Partial and complete remission rates and glucocorticoid-related toxicity were assessed. Results: $15 \mathrm{CPC}$ and $30 \mathrm{HC}$ patients were analysed. The mean (SD) initial dose of prednisone was $22(8) \mathrm{mg} / \mathrm{d}$ in the CPC vs. 49 (19) $\mathrm{mg} / \mathrm{d}$ in the $\mathrm{HC}(\mathrm{p}<0.001)$. The 6-month mean (SD) cumulative dose of prednisone was $1.7(0.5) \mathrm{g}$ (average daily dose $9 \mathrm{mg}$ ) vs. $4.5(2.1) \mathrm{g}$ (average daily dose $25 \mathrm{mg}$ ), respectively $(\mathrm{p}<0.001)$. The median cumulative dose of cyclophosphamide at six months was $3(0-4.5) \mathrm{g}$ in the CPC vs. $5(0-16.8)$ in the HC (p<0.001). 15/15 $(100 \%)$ vs. $10 / 30(33 \%)$ patients were treated with hydroxychloroquine $(\mathrm{p}<0.001)$. At six months, $12 / 15(80 \%)$ patients in the CPC achieved partial or complete remission vs. 14/30 (47\%) in the HC $(\mathrm{p}=0.015)$. At 12 months, $13 / 15(87 \%)$ vs. $19 / 30(63 \%)$ patients, respectively, were in complete or partial remission $(\mathrm{p}=0.055)$. Toxicity attributable to glucocorticoids was observed in $1 / 15(7 \%)$ vs. $20 / 30(67 \%)$ patients, respectively $(\mathrm{p}<0.0001)$. Conclusion: A combination of medium-dose prednisone, methylprednisolone pulses, cyclophosphamide and hydroxychloroquine is at least as effective in achieving remission of lupus nephritis as regimes containing high-dose prednisone and causes less toxicity
\end{abstract}

Key Words: Systemic lupus erythematosus; Glucocorticoids; Hydroxychloroquine; Adverse effects; Osteonecrosis 


\title{
Multinational Evidence-Based Recommendations for the Diagnosis and Management of Gout
}

\section{Francisca Sivera et al.}

Ann Rheum Dis 2014; 73: 328-335

\begin{abstract}
We aimed to develop evidence-based multinational recommendations for the diagnosis and management of gout. Using a formal voting process, a panel of 78 international rheumatologists developed 10 key clinical questions pertinent to the diagnosis and management of gout. Each question was investigated with a systematic literature review. Medline, Embase, Cochrane CENTRAL and abstracts from 2010-2011 European League Against Rheumatism and American College of Rheumatology meetings were searched in each review. Relevant studies were independently reviewed by two individuals for data extraction and synthesis and risk of bias assessment. Using this evidence, rheumatologists from 14 countries (Europe, South America and Australasia) developed national recommendations. After rounds of discussion and voting, multinational recommendations were formulated. Each recommendation was graded according to the level of evidence. Agreement and potential impact on clinical practice were assessed. Combining evidence and clinical expertise, 10 recommendations were produced. One recommendation referred to the diagnosis of gout, two referred to cardiovascular and renal comorbidities, six focused on different aspects of the management of gout (including drug treatment and monitoring), and the last recommendation referred to the management of asymptomatic hyperuricaemia. The level of agreement with the recommendations ranged from 8.1 to 9.2 (mean 8.7) on a 1-10 scale, with 10 representing full agreement. Ten recommendations on the diagnosis and management of gout were established. They are evidence-based and supported by a large panel of rheumatologists from 14 countries, enhancing their utility in clinical practice.
\end{abstract}

\section{A systematic Review of Recommendations and Guidelines for the Management of Osteoarthritis}

\author{
Amanda E. Nelson et al. \\ Seminars in Arthritis and Rheumatism, published online 05 December 2013
}

Purpose: Although a number of osteoarthritis (OA) management guidelines exist, uptake has been suboptimal. Our aim was to review and critically evaluate existing OA management guidelines to better understand potential issues and barriers. Methods: A systematic review of the literature in MEDLINE published from January 1, 2000 to April 1, 2013 was performed and supplemented by bibliographic reviews, following PRISMA guidelines and a written protocol. Following initial title and abstract screening, 2 authors independently reviewed full-text articles; a third settled disagreements. Two independent reviewers extracted data into a standardized form. Two authors independently assessed guideline quality using the AGREE II instrument; three generated summary recommendations based on the extracted guideline data. Results: Overall, 16 articles were included in the final review. There was broad agreement on recommendations by the various organizations. For non-pharmacologic modalities, education/self-management, exercise, weight loss if overweight, walking aids as indicated, and thermal modalities were widely recommended. For appropriate patients, joint replacement was recommended; arthroscopy with debridement was not recommended for symptomatic knee OA. Pharmacologic modalities most recommended included acetaminophen/paracetamol (first line) and NSAIDs (topical or oral, second line). Intra-articular corticosteroids were generally recommended for hip and knee OA. Controversy remains about the use of acupuncture, knee braces, heel wedges, intra-articular hyaluronans, and glucosamine/chondroitin. Conclusions: The relative agreement on many OA management recommendations across organizations indicates a problem with dissemination and implementation rather than a lack of quality guidelines. Future efforts should focus on optimizing implementation in primary care settings, where the majority of OA care occurs.

Key Words: Osteoarthritis, Management, Guidelines, Systematic review

Egypt J Rheumatology and Clinical Immunology | Jan 2014 | Vol. 2 | Issue 1 


\title{
International Recommendations for the Assessment of Autoantibodies to Cellular Antigens Referred to as Anti-nuclear Antibodies (ANA)
}

\author{
Agmon-Levin N, et al.
}

Ann Rheum Dis 2014; 73: 17-23.

\begin{abstract}
Anti-nuclear antibodies (ANA) are fundamental for the diagnosis of autoimmune diseases, and have been determined by indirect immunofluorescence assay (IIFA) for decades. As the demand for ANA testing increased, alternative techniques were developed challenging the classic IIFA. These alternative platforms differ in their antigen profiles, sensitivity and specificity, raising uncertainties regarding standardisation and interpretation of incongruent results. Therefore, an international group of experts has created recommendations for ANA testing by different methods. Two groups of experts participated in this initiative.The European autoimmunity standardization initiative representing 15 European countries and the International Union of Immunologic Societies/World Health Organization/Arthritis Foundation/Centers for Disease Control and Prevention autoantibody standardizing committee. A three-step process followed by a Delphi exercise with closed voting was applied.Twenty-five recommendations for determining ANA(113), anti-double stranded DNA antibodies (14-18), specific antibodies (19-23) and validation of methods(24-25) were created. Significant differences between experts were observed regarding recommendations 24-25 ( $\mathrm{p}<0.03)$. Here, we formulated recommendations for the assessment and interpretation of ANA and associated antibodies. Notably, the roles of IIFA as a reference method, and the importance of defining nuclear and cytoplasmic staining, were emphasised, while the need to incorporate alternative automated methods was acknowledged. Various approaches to overcome discrepancies between methods were suggested of which an improved bench-to-bedside communication is of the utmost importance. These recommendations are based on current knowledge and can enable harmonisation of local algorithms for testing and evaluation of ANA and related autoantibodies. Last but not least, new more appropriate terminologies have been suggested.
\end{abstract}

\section{Discontinuation of Adalimumab after Achieving Remission in Patients with Established Rheumatoid Arthritis: 1-Year Outcome of the HONOR Study}

\author{
Tanaka Y, et al. \\ Ann Rheum Dis 2013; 0:1-7.
}

Objectives: To investigate the possibility of discontinuing adalimumab (ADA) for 1 year without flaring (DAS28erythrocyte sedimentation rate $(\mathrm{ESR}) \geq 3.2$ ), and to identify factors enabling established patients with rheumatoid arthritis (RA) to remain ADA-free. Methods: Of 197 RA patients treated with ADA +methotrexate (MTX), 75 patients who met the ADA-free criteria (steroid-free and sustained DAS28-ESR remission for 6 months with stable MTX doses) were studied for 1 year. Results: The mean disease duration and DAS28-ESR score in 75 patients was 7.5 years and 5.1 at baseline, respectively. The proportion of patients who sustained DAS28-ESR $<2.6(48 \%)$ and DAS28-ESR $<3.2(62 \%)$ for 1 year were significantly lower in the ADA discontinuation group than in the ADA continuation group; however, in patients with deep remission (DAS28-ESR $\leq 1.98$ ) identified by receiver operating characteristics analysis following logistic analysis, these rates increased to $68 \%$ and $79 \%$, respectively, with no significant difference between both groups. Remarkably, ADA readministration to patients with flare was effective in returning DAS28-ESR to $<3.2$ within 6 months in $90 \%$ and 9 months in $100 \%$ patients; among the patients who sustained DAS28-ESR <3.2 during ADA discontinuation, 100\% remained in structural remission and 94\% in functional remission. Conclusions: The possibility of remaining ADA-free for 1 year was demonstrated in established patients with RA with outcomes that ADA can be discontinued without flaring in 79\% patients with deep remission, with similar rates in the ADA continuation group, and showed no functional or structural damage in patients with DAS28- ESR <3.2. ADA readministration to patients with flare during ADA discontinuation was effective. 